\title{
Tolérance immunitaire : délétion clonale et anergie
}

Les mécanismes de la tolérance immunitaire aux antigènes du self ont été plusieurs fois discutés dans ces colonnes (m/s suppl. au $n^{\circ} 1$, vol. 5 , p. 25 et $n^{\circ} 10$, vol. 5 , p. 788). Ils mettent avant tout en jeu un processus de délétion clonale des clones autoréactifs au cours de l'éducation dans le thymus des lymphocytes $T$. Cette sélection négative survient à un stade tardif de la maturation des thymocytes dans le cortex thymique, peut-être au niveau des cellules dendritiques situées à l'interface médulla/cortex. Un récent article de chercheurs du NIH à Bethesda (USA) indique que, chez des souris receveuses irradiées (dont le thymus contient donc des cellules stromales, radiorésistantes, alors que les cellules d'origine médullaire ont été détruites), la délétion clonale de cellules médullaires greffées reconnaissant un antigène de l'hôte ne survient pas [1]. Ces résultats indiquent donc que le processus de délétion clonale nécessite probablement que l'interaction des cellules autoréactives se produise avec un antigène du self présenté par des cellules d'origine médullaire, et non par des cellules stromales. Cependant, les animaux chimères ainsi constitués, qui n'ont pas éliminé leurs clones autoréactifs, n'ont néanmoins pas de symptôme d'atteinte auto-immune. De fait, les clones potentiellement autoréactifs ne sont pas stimulés pär l'antigène. Ce phénomène de tolérance sans délétion clonale est appelé « anergie » [2]. Ses bases moléculaires restent mystérieuses, mais certains travaux récents indiquent qu'elles pourraient inclure la nécessité d'un deuxième signal qui dépendrait du type de contact entre la cellule $\mathrm{T}$ et l'antigène. En d'autres termes, lorsqu'une cellule $T$ spécifique d'un antigène (par exemple du self) le rencontrerait, présenté par une cellule non spécialisée dans la présen- en serait une anergie profonde et prolongée [3]. Un tel phénomène expliquerait un important paradoxe : la «suppression» de clones autoréactifs dirigés contre des antigènes du self dont on suppose qu'ils ne sont jamais synthétisés dans le thymus, soit parce que leur spécificité tissulaire exclut le thymus, soit parce qu'ils sont de toute façon synthétisés tardivement au cours du développement fœetal, bien après la phase d'apprentissage thymique des lymphocytes $T$. On peut imaginer alors que ces cellules autoréactives non délétées reconnaissant, dans l'organisme mature, un antigène présenté, par exemple, par un organe particulier, seraient rendues anergiques. C'est à une conclusion de ce type que sont également parvenues plusieurs équipes américaines associées (Biogen, Cambridge, MA ; Philadelphia, PA ; Lilly Res. Lab., La Jolla, CA ; Yale, New Haven, CT, Scripps Clinic, La Jolla, CA) en étudiant des souris transgéniques exprimant un gène codant pour une molécule du CMH de classe II sous le contrôle soit du promoteur du gène de l'insuline, soit sous celui du gène de l'élastase. Dans les deux cas existent des populations de lymphocytes $T$ potentiellement autoréactifs mais répondant peu ou pas à la stimulation par des anticorps antichaîne $\beta$ du récepteur T (TcR) [4]. Cette anergie peut être rapprochée de l'expression de la molécule de classe II transgénique par des cellules non spécialisées, en l'occurence le pancréas endocrine ou exocrine. En revanche, des cellules qui rencontreraient un antigène dont elles sont spécifiques, présenté par des cellules spécialisées dans le « traitement » des antigènes étrangers (macrophages, cellules dendritiques, lymphocytes $B)$, en recevraient le «deuxième signal » nécessaire à l'induction de la prolifération. Le même mécanisme s'applique probablement aux cel- lules B autoréactives qui pourraient, au cours du développement, être l'objet d'une délétion clonale et, dans les tissus périphériques, chez le foetus aussi bien que chez l'adulte, être « inactivées » [5]. On peut supposer ici que le «deuxième signal », nécessaire à une réponse proliférative et dont l'absence aboutit à l'anergie, est constitué par la présence de cellules $T$ auxiliaires actives et spécifiques de l'antigène (cellules $\mathrm{T}$ helper) ( $m / s$ suppl. au $n^{\circ} 7$, vol. 5 , p. 1 et p. 14). Un antigène du self apparaissant tardivement et n'entraînant pas de réponse lymphocytaire $\mathrm{T}$ provoquera également une anergie des cellules B. Une équipe australienne de Sydney vient de montrer que cette transition de cellules $\mathrm{B}$ autoréactives vers l'anergie, provoquée par la rencontre de l'auto-antigène en l'absence de cellules $T$ auxiliaires, s'accompagne d'une modification de l'expression des immunoglobulines de membrane [5] : l'IgM, prédominante au niveau des cellules réactives, est en grande partie remplacée par de l'IgD par une modification d'épissage du transcrit primitif IgM/D ( $\mathrm{m} / \mathrm{s}$ suppl. au $n^{\circ} 1$, vol. 5 , p. 5). Tous ces récents résultats complètent peu à peu notre compréhension des mécanismes de discrimination entre le self et l'étranger.

A. $\mathbf{K}$.

1. Ramsdell F, Lantz T, Fowlkes BJ. A nondeletional mechanism of thymic self tolerance. Science $1989 ; 246$ : 1038-41.

2. Mac Donald HR. Mechanisms of immunological tolerance. Science $1989 ; 246$ : 982. 3. De France AL. Tolerance: a second mechanism. Nature $1989 ; 342: 340-1$.

4. Burkly LC, Lo D, Kanagawa O, Brinster RL, Flavell RA. T-cell tolerance by clonal anergy in transgenic mice with nonlymphoid expression of MHC class II I-E. Nature 1989; 342: $564-6$.

5. Goodnow CC, Crosbie J, Jorgensen H, Brink RA, Basten A. Induction of self tolerance in mature peripheral B lymphocytes. Nalure $1989 ; 342$ : 385-90. 